\title{
Synchronous and Asynchronous Sequential Symbol Synchronizers
}

\author{
António D. Reis ${ }^{1,2}$, José F. Rocha ${ }^{1}$, Atilio S. Gameiro ${ }^{1}$, José P. Carvalho ${ }^{2}$ \\ ${ }^{1}$ Dep. de Electrónica e Telecomunicações / Instituto de Telecomunicações, Universidade de Aveiro, 3810 Aveiro, Portugal \\ ${ }^{2}$ Dep. de Fisica / U. D. Remota, Universidade da Beira Interior Covilhã, 6200 Covilhã, Portugal
}

\begin{abstract}
In this work, we present two synchronizer groups: the synchronous and the asynchronous.

The synchronous group is based in forward logic with flip flops and the asynchronous group is based in forward logic with delay line feedback.

In each group we consider two versions: the manual and the automatic.

The main objective is to study the two groups, each one with two versions and to observe its jitter performance as function of the noise.
\end{abstract}

Key words: Synchronism in Digital Communications

\section{INTRODUCTION}

The final system quality depends strongly of the synchronizer performance $[1,2,3,4,5,6,7,8,9]$.

The synchronizer samples the data at the optimum point and retimes the bit duration $[10,11,12,13,14,15,16]$.

There is a great variety of synchronizers, but here we go present a prototype based on the comparison between a variable pulse area $\mathrm{Pv}$ and a fixed pulse area Pf. The area difference $\mathrm{Pr}$ is the error signal, that corrects the position of the VCO (Voltage Controlled Oscillator) toward the optimum point. This point is the maximum opening eye diagram and corresponds normally to the bit center.

The following figure (Fig.1) shows the configuration of the synchronizer based in pulses area comparison.

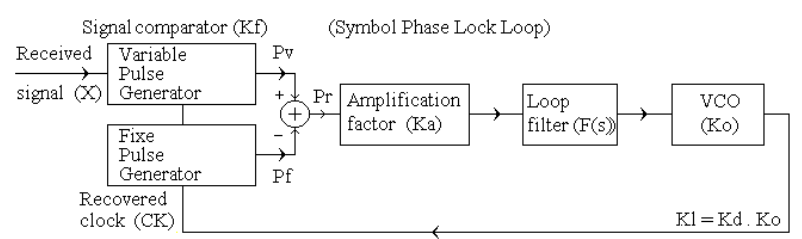

Fig.1 Configuration of the synchronizer based on two pulses (Pv,Pf)

The $\mathrm{Kf}$ is the signal (phase / frequency) comparator gain, $\mathrm{F}(\mathrm{s})$ is the loop filter, $\mathrm{Ko}$ is the $\mathrm{VCO}$ gain and $\mathrm{Ka}$ is the control parameter of the loop gain, that acts in the locus root providing the desired characteristics.

We will implement the above blocks based in two different technologies originating two distinct groups which are the synchronous and the asynchronous. The synchronous is based in logic with flip flops and the asynchronous is based in logic with feedback.

\footnotetext{
${ }^{12} \mathrm{UA}-\mathrm{UBI}$
}

The following figure (Fig.2) illustrates the basic difference between a synchronous and asynchronous system.
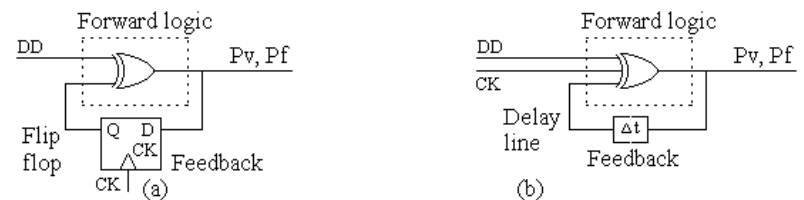

Fig.2 Aspect of the synchronous (a) and asynchronous (b) type

Each group has two versions, which are the manual and the automatic.

In the manual version, the fixed pulse generation is based in a delay line that needs human adjusting. Oppositly, in the automatic version the fixed pulse generation is based in a flip flop dispensing the human adjusting.

The following figure (Fig.3) illustrates the basic difference between the manual and automatic versions. (a)

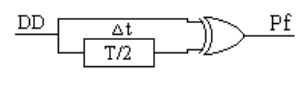

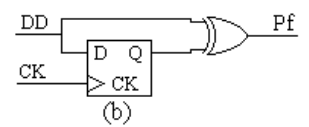

Fig.3 Aspect of the manual (a) and automatic (b) version

Next, we present separately, the two groups of synchronizers, firstly the synchronous synchronizers and after the asynchronous synchronizers. Each group has two versions which are the manual and automatic.

After, we test the two groups with their two versions manual and automatic.

Then, we present the results and we make some comparisons.

Finally, we present some conclusions.

\section{SYNCHRONOUS SYNCHRONIZERS}

We begin presenting the synchronous synchronizer based on flip flops with logic. We distinguish the manual and the automatic versions [1, 2, 3, 4].

Fig. 4 shows the synchronous type manual version

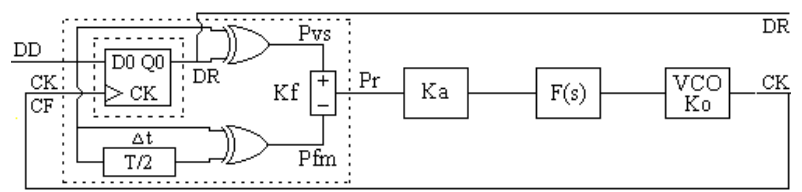

Fig.4 Manual synchronous symbol synchronizer (tx1sm)

The manual version is based on the comparation between the phase comparator Pvs and manual reference pulse Pfm. 
Fig. 5 shows the synchronous type automatic version

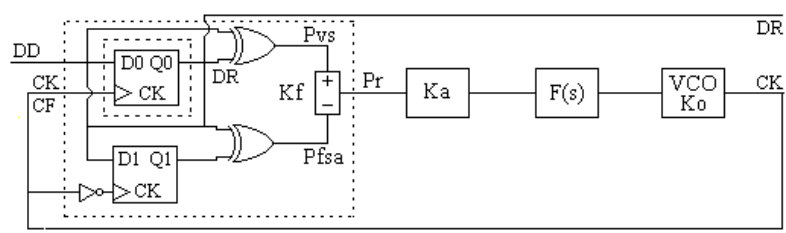

Fig.5 Automatic synchronous symbol synchronizer (tx1sa)

The automatic version is based on the comparation between the phase comparator Pvs and the automatic reference pulse Pfsa.

\section{ASYNCHRONOUS SYNCHRONIZERS}

Now, we present the asynchronous synchronizers based on logic with delay line feedback. We distinguish the manual and the automatic versions $[6,7,8,9,10]$.

Fig. 6 shows the asynchronous type manual version.

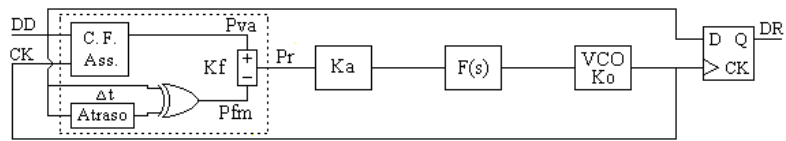

Fig.6 Manual asynchronous symbol synchronizer (tx1am)

The manual version is based on the comparation between the asynchronous phase comparator Pva and the manual reference pulse Pfm.

Fig.7 shows the asynchronous type automatic version.

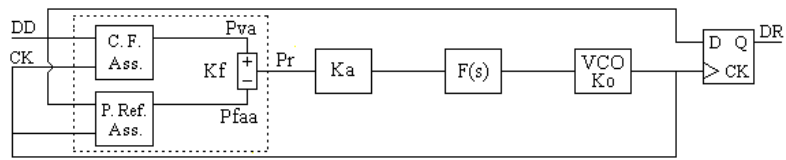

Fig.7 Automatic asynchronous symbol synchronizer (tx1aa)

The automatic version is based on the comparation between the asynchronous phase comparator Pva and the automatic reference pulse Pfaa.

Fig. 8 shows the implementation of the asynchronous phase comparator Pva.

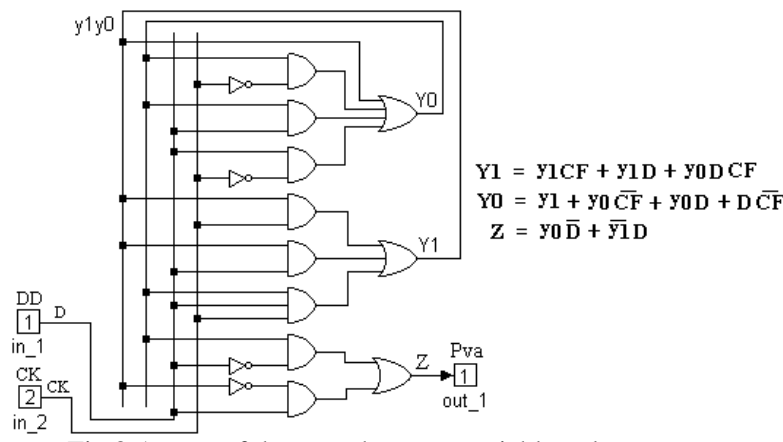

Fig.8 Aspect of the asynchronous variable pulse generator

Fig.9 shows the implementation of the asynchronous reference pulse Pfaa.

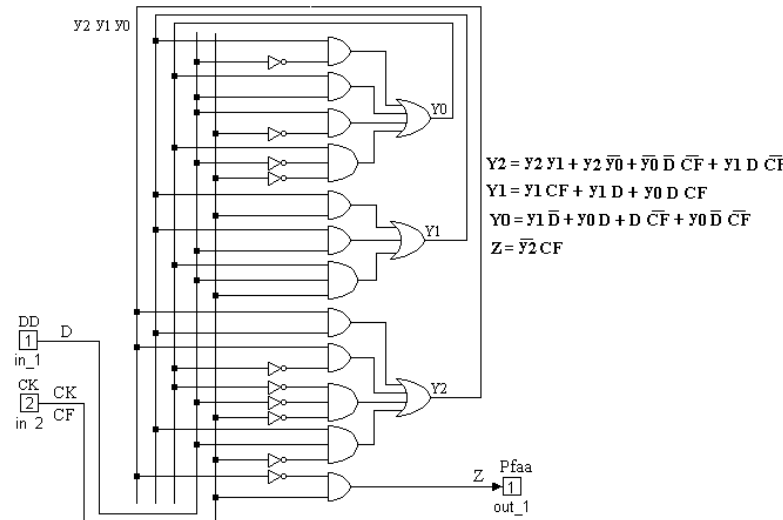

Fig.9 Aspect of the asynchronous fixed pulse generator

This asynchronous phase comparator and reference pulse was projected by asynchronous methods.

\section{TESTS, DESIGN AND RESULTS}

We will present the tests, the design and the results of the referred synchronizers [5].

\section{A. Tests}

The following figure (Fig.10) shows the setup that was used to test the various synchronizers.

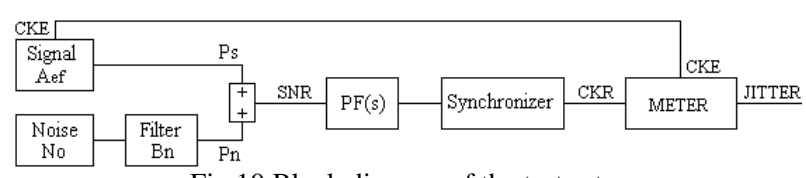

Fig.10 Block diagram of the test setup

The receiver recovered clock with jitter is compared with the emitter original clock without jitter, the difference is the jitter of the received clock.

\section{B. Jitter measurer (Meter)}

The jitter measurer (Meter) consists of a RS flip flop, which detects the random variable phase of the recovered clock (CKR), relatively to the fixed phase of the emitter clock (CKE).

This relative random phase variation is the recovered clock jitter (Fig.11).

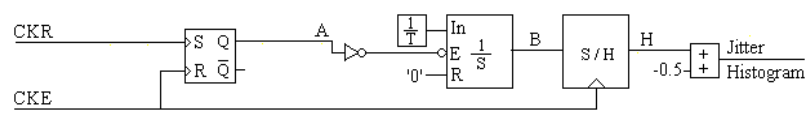

Fig.11 The jitter measurer (Meter)

The other blocks convert this random phase variation into a random amplitude variation, which is the jitter histogram.

Then, the jitter histogram is sampled and processed by an appropriate program, providing the RMS jitter and the peak to peak jitter. 


\section{Design}

To get guaranteed results, it is necessary to dimension all the synchronizers with equal conditions. Then it is necessary to design all the loops with identical linearized transfer functions.

The general loop gain is $\mathrm{Kl}=\mathrm{Kd} . \mathrm{Ko}=\mathrm{Ka} . \mathrm{Kf}$.Ko where $\mathrm{Kf}$ is the phase comparator gain, $\mathrm{Ko}$ is the $\mathrm{VCO}$ gain and $\mathrm{Ka}$ is the amplification control factor that permits the desired characteristics.

For analysis facilities, we use a normalized transmission rate $t x=1$ baud, what implies also normalized values for the others dependent parameters. So, the normalized clock frequency is $\mathrm{fCK}=1 \mathrm{~Hz}$.

We choose a normalized external noise bandwidth $\mathrm{Bn}=$ $5 \mathrm{~Hz}$ and a normalized loop noise bandwidth $\mathrm{Bl}=0.02 \mathrm{~Hz}$. Later, we can disnormalize these values to the appropriated transmission rate tx.

Now, we will apply a signal to noise ratio SNR related with the signal amplitude Aef, noise spectral density No and external noise bandwidth $\mathrm{Bn}$, so it is $\mathrm{SNR}=\mathrm{A}_{\mathrm{ef}}^{2} /(\mathrm{No} . \mathrm{Bn})$. But No can be related with the noise variance on and inverse sampling $\Delta \tau=1 /$ Samp, then $\quad \mathrm{No}=2 \sigma \mathrm{n}^{2} . \Delta \tau$, so $\mathrm{SNR}=\mathrm{A}_{\mathrm{ef}}^{2} /\left(2 \sigma \mathrm{n}^{2} . \Delta \tau \cdot \mathrm{Bn}\right)=0.5^{2} /\left(2 \sigma \mathrm{n}^{2} * 10^{-3} * 5\right)=25 / \sigma \mathrm{n}^{2}$.

\section{- $1^{\text {st }}$ order loop:}

The loop filter $\mathrm{F}(\mathrm{s})=1$ with cutoff frequency $0.5 \mathrm{~Hz}(\mathrm{Bp}=0.5$ $\mathrm{Hz}$ is 25 times bigger than $\mathrm{Bl}=0.02 \mathrm{~Hz}$ ) eliminates only the high frequency, but maintain the loop characteristics.

The transfer function is

$$
H(s)=\frac{\mathrm{G}(\mathrm{s})}{1+\mathrm{G}(\mathrm{s})}=\frac{K d K o F(s)}{s+K d K o F(s)}=\frac{K d K o}{s+K d K o}
$$

the loop noise bandwidth is

$$
\mathrm{Bl}=\frac{K d K o}{4}=K a \frac{K f K o}{4}=0.02 \mathrm{~Hz}
$$

Then, for analog synchronizers, the loop bandwidth is $B l=0.02=(K a . K f . K o) / 4$ with $(\mathrm{Km}=1, \mathrm{~A}=1 / 2, \mathrm{~B}=1 / 2 ; \mathrm{Ko}=2 \pi)$

$($ Ka.Km.A.B.Ko $) / 4=0.02->K a=0.08 * 2 / \pi$

For hybrid synchronizers, the loop bandwidth is $B l=0.02=(K a \cdot K f \cdot K o) / 4$ with $(\mathrm{Km}=1, \mathrm{~A}=1 / 2, \mathrm{~B}=0.45 ; \mathrm{Ko}=2 \pi)$

$($ Ka.Km.A.B.Ko $) / 4=0.02->K a=0.08 * 2.2 / \pi$

For combinational synchronizers, the loop bandwidth is $B l=0.02=(K a . K f . K o) / 4 \quad$ with $\quad(\mathrm{Kf}=1 / \pi ; \mathrm{Ko}=2 \pi)$

$\left(K a * 1 / \pi^{*} 2 \pi\right) / 4=0.02->K a=0.04$

For sequential synchronizers (present case), the loop bandwidth is

$B l=0.02=(K a \cdot K f . K o) / 4 \quad$ with $\quad(\mathrm{Kf}=1 / 2 \pi ; \mathrm{Ko}=2 \pi)$

$(K a * 1 / 2 \pi * 2 \pi) / 4=0.02->K a=0.08$
The jitter depends on the RMS signal Aef, on the power spectral density No and on the loop noise bandwidth Bl.

For analog PLL the jitter is

$\sigma \phi^{2}=B l . N o / A e f^{2}=$ B1.2. $\sigma n^{2} . \Delta \tau=0.02 * 10^{-3} * 2 \sigma n^{2} / 0.5^{2}=16^{*} 10^{-5} . \sigma n^{2}$

For the others PLLs the jitter formula is more complicated.

\section{$-2^{\text {nd }}$ order loop:}

The second order loop is not shown here, but the results are identical to the ones obtained above for the first order loop.

\section{Results}

We present, the synchronizers jitter behavior as function of the noise.

Fig. 12 shows the jitter - noise curves of the four synchronizers (tx1sm, tx1sa, tx1am, tx1aa).

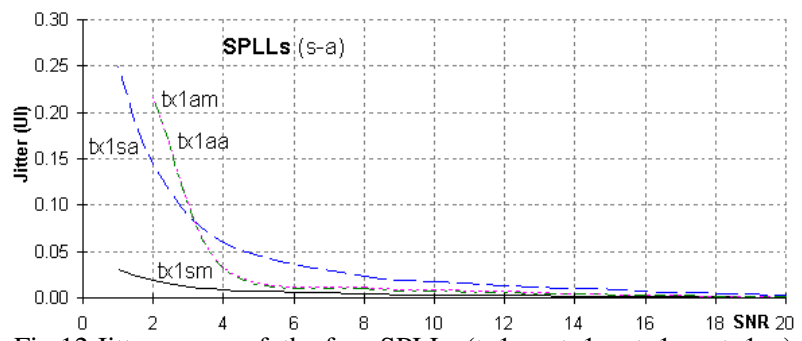

Fig. 12 Jitter curves of the four SPLLs (tx1sm, tx1sa, tx1am, tx1aa)

We note that generically in all the synchronizers the jitter UIRMS diminishes when the signal to noise ratio SNR increases.

We verify that the manual synchronous is always the best case for all SNR. For SNR>3 when all synchronizers are in lock, the automatic synchronous is the worst case and the two asynchronous prototypes have intermedium similar behavior. For $\mathrm{SNR}<3$ the asynchronous prototypes lost the lock, causing a great increment of the jitter.

\section{CONCLUSIONS}

We studied four Sequential Symbol Synchronizers (tx1sm, tx1sa, tx1am, tx1aa).

The results show that the synchronous prototypes have different behavior but the asynchronous have equal behavior.

For SNR $>3$ with all synchronizers in perfect lock the manual synchronous is the best case and the automatic synchronous the worst case. The asynchronous prototypes have an intermediate behavior. For $\mathrm{SNR}<3$ the two asynchronous prototypes, begin with synchronism problems and the jitter increases suddenly. This is caused by noise that passes through the gates and provokes races in the circuits.

This is comprehensible since the output jitter depends on the input noise and the error state of the phase comparator. In the synchronous manual only the phase comparator is sequential (memory) and has contribution to error state, the reference pulse is combinational.

In the synchronous automatic, the phase comparator is sequential causing error state, the reference pulse is also sequential causing error state and there is still the error propagation effect from the first to the second. 
In the asynchronous manual and automatic cases, the phase comparator and reference pulse are projected independently. Then each one has its own error state, but is avoid the error propagation from the first to the second. Then this results in an intermedium jitter behavior. The phase comparator is more opened to the noise than the reference pulse, then its effect is predominant and the jiiter is equal in the two cases.

\section{ACKNOWLEDGMENTS}

The authors are grateful to the program FCT (Foundation for Science and Technology).

\section{REFERENCES}

[1] A. H. Jazwinski, "Filtering for Nonlinear Dynamical Systems" IEEE Tra. Automatic Control p.765 Oct. 1966.

[2] J. C. Imbeaux, "performance of the delay-line multiplier circuit for clock and carrier synchronization", IEEE Jou. on Selected Areas in Communications p.82 Jan. 1983.

[3] Werner Rosenkranz, "Phase Locked Loops with limiter phase detectors in the presence of noise", IEEE Trans. on Communications com-30 No10 pp.2297-2304. Oct 1982.

[4] Charles R. Hogge, "A Self Correcting Clock Recovery Circuit”, IEEE Tran. Electron Devices p.2704 Dec 1985.

[5] A. D. Reis, J. F. Rocha, A. S. Gameiro, J. P. Carvalho "A New Technique to Measure the Jitter", Proc. III Conf. on Telecommunications pp.64-67 FFoz-PT 23-24 Apr 2001.

[6] Marvin K. Simon, William C. Lindsey, "Tracking Performance of Symbol Synchronizers for Manchester Coded Data", IEEE Transactions on Communications Vol. com-2.5 No4, pp.393-408, April 1977.

[7] Jung-Hui Chiu, Lin-Shan Lee "The Minimum Likelihood - A New Concept for Bit Synchronization", IEEE Transactions on Communications Vol. com-95 N 5 , pp.545-549, May 1987.

[8] J. B. Carruthers, D. D. Falconer, H. M. Sandler, L. Strawczynski, "Bit Synchronization in the Presence of Co-Channel Interference", Proc. Canadian Conference on Electrical and Computer Engineering pp.4.1.1-4.1.7, Ottawa, Canada, 3-6 Sep. 1990.

[9] Johannes Huber, Weilin Liu "Data-Aided Synchronization of Coherent CPM-Receivers" IEEE Transactions on Communications Vol.40 Nº pp.178189, Jan. 1992.

[10] K. Bucket, M. Moeneclaey, "The Effect of Non-Ideal Interpolation on Symbol Synchronization Performance", Proc. 3rd European Confer. on Satellite Communication pp.379-383, Manchester-UK, 2-4 Nov. 1993.

[11] Antonio A. D'Amico, Aldo N. D'Andrea, Ruggero Reggianni, "Efficient Non-Data-Aided Carrier and Clock Recovery for Satellite DVB at Vary Low Signalto-Noise Ratios", IEEE Journal on Satellite Areas in Communications Vol.19 Nº12 pp.2320-2330, Dec. 2001.

[12] Yimin Jiang, Feng-Wen Sun, John S. Baras, "On the Performance Limits of Data-Aided Synchronization", IEEE Transaction on Information Theory Vol. $49 \mathrm{~N}^{\mathrm{o}} 1$, pp.191-203, Jan 2003.
[13] Rostislav Dobkin, Ran Ginosar, Christos P. Sotiriou "Data Synchronization Issues in GALS SoCs", Proc. 10th International Symposium on Asynchronous Circuits and Systems, pp.CD-Ed., Crete-Greece 19-23 Apr. 2004.

[14] M. J. Canet, I. J. Wassell, J. Valls, V. Almenar, "Performance Evaluation of Fine Time Synchronizers for WLANS", Proc. 13th European Signal Processing Conference, pp.CD-Edited, Antalya-Turk. 4-8 Sep. 2005.

[15] N. Noels, H. Steendam, M. Moeneclaey, "Effectiveness Study of Code-Aided and Non-Code-Aided ML-Based Feedback Phase Synchronizers", Proc. IEEE International Conference on Communications (ICC'06) pp.2946-2951, Istambul-Turkey, 11-15 June 2006.

[16] A. D. Reis, J. F. Rocha, A. S. Gameiro, J. P. Carvalho "Synchronizers Operating by Two or One Data Transitions", Proc. V Symposium on Enabling Optical Network and Sensors (SEONs 2007) p9.87-88, AveiroPT 29-29 June 2007. 